\title{
Structural characterization and evaluation of prebiotic activity of oil palm kernel cake mannanoligosaccharides
}

\begin{abstract}
In this study, mannanoligosaccharides (MOS) were isolated from palm kernel cake by aqueous extraction using high temperature and pressure. Structural characterization of MOS was carried out using acid hydrolysis, methylation analysis, ESI-MS/MS and 1D/2D NMR. The prebiotic activity of MOS was evaluated in vitro using two probiotic Lactobacillus strains. Sugar analysis indicated the presence of mannose in each of the oligomers. Methylation and 1D/2D NMR analysis indicated that the MOS have a linear structure consisting of $(1 \rightarrow 4)-\beta$-d-mannopyranosyl residues. ESI-MS/MS results showed that the isolated mannan oligomers, MOS-III, MOS-IV, MOS-V and MOS-VI consist of tetra-, penta-, hexa-, and hepta-saccharides with molecular weights of 689, 851, 1013 and $1151 \mathrm{Da}$, respectively. Based on the in vitro growth study, MOS-III and MOS-IV was found to be effective in selectively promoting the growth of Lactobacillus reuteri $\mathrm{C} 1$ strain as evidenced by the optical density of the culture broth.
\end{abstract}

Keyword: Mannanoligosaccharides; Palm kernel cake; ESI-MS/MS; 1D/2D NMR experiments; Prebiotic activity 
